
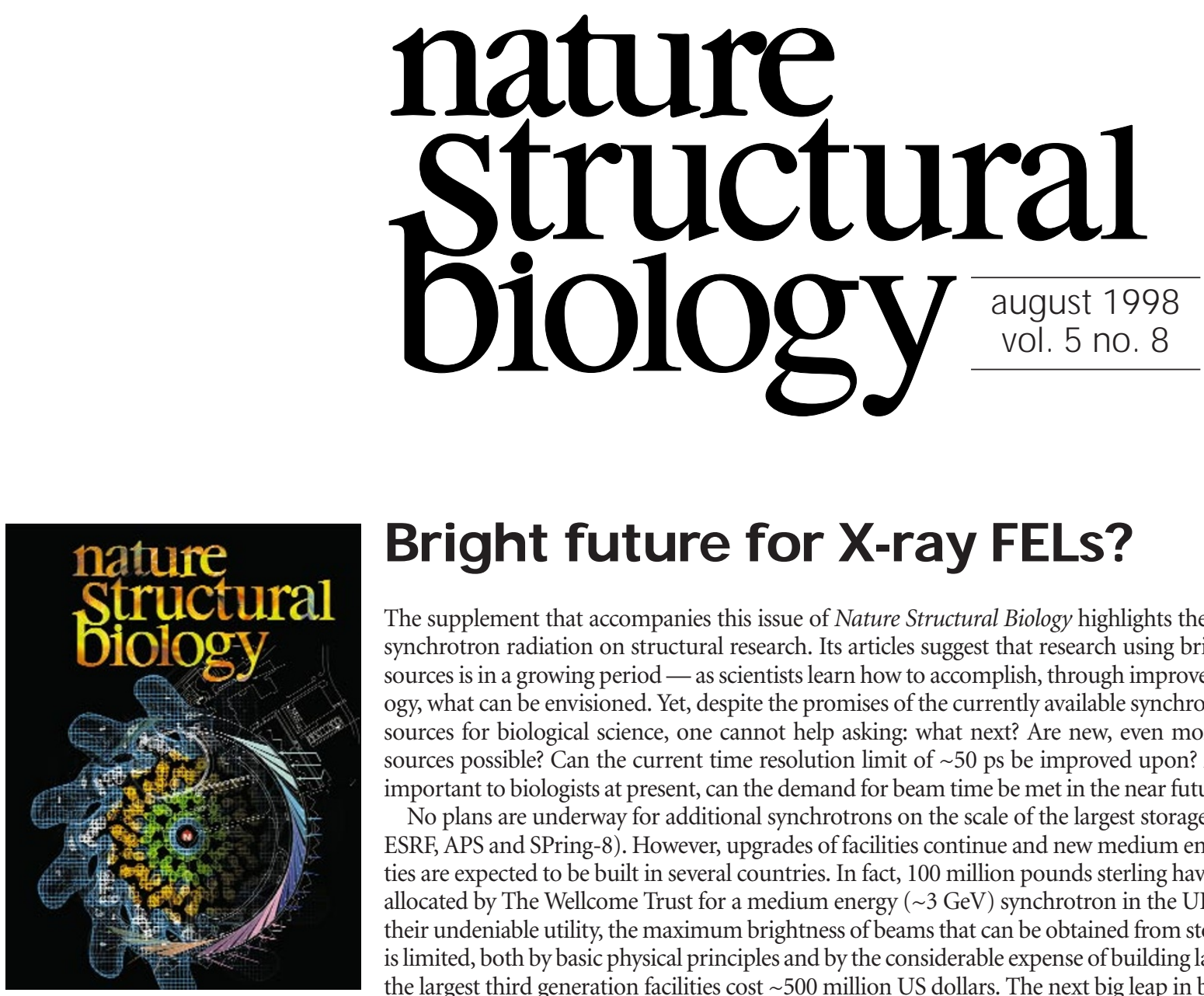

\title{
Bright future for X-ray FELs?
}

The supplement that accompanies this issue of Nature Structural Biology highlights the impact of synchrotron radiation on structural research. Its articles suggest that research using brilliant light sources is in a growing period - as scientists learn how to accomplish, through improved technology, what can be envisioned. Yet, despite the promises of the currently available synchrotron X-ray sources for biological science, one cannot help asking: what next? Are new, even more brilliant sources possible? Can the current time resolution limit of $\sim 50$ ps be improved upon? And, most important to biologists at present, can the demand for beam time be met in the near future?

No plans are underway for additional synchrotrons on the scale of the largest storage rings (the ESRF, APS and SPring-8). However, upgrades of facilities continue and new medium energy facilities are expected to be built in several countries. In fact, 100 million pounds sterling have just been allocated by The Wellcome Trust for a medium energy $(\sim 3 \mathrm{GeV})$ synchrotron in the UK ${ }^{1}$. Despite their undeniable utility, the maximum brightness of beams that can be obtained from storage rings is limited, both by basic physical principles and by the considerable expense of building larger rings; the largest third generation facilities cost $\sim 500$ million US dollars. The next big leap in brilliance is likely to come instead from a free electron laser (FEL; pronounced F, E, L) that will produce coherent radiation in the X-ray wavelength range (and is thus often termed an XFEL).

What is an FEL, and how would radiation from an XFEL differ from synchrotron radiation? In simple terms, an FEL is a laser (a source of highly coherent radiation) that can be realized when a very compact pulse of high-energy electrons produces radiation in an undulator (a set of magnets of alternating polarity). The intense undulator radiation bathes the electrons traveling at relativistic speeds, causing them to bunch together in regions the size of the radiation wavelength. When this occurs, the electrons act coherently, giving increased emission, which in turn gives increased bunching and an exponential rise in output power down the length of the undulator. This is in contrast to the situation in a typical synchrotron source where the electron bunches are not as compact and do not behave coherently; in that case, the radiation emitted increases only linearly with the length of the undulator. The peak brightness of an XFEL could be more than ten orders of magnitude greater than that of third-generation synchrotron sources (Fig. 1). In addition to the huge increase in brightness, the XFEL would produce very short X-ray pulses, allowing time resolution in the fs range, hundreds of times faster than can be achieved presently with synchrotrons. Such a tremendous leap in performance would clearly herald the coming of a new generation of X-ray sources. These sources would look very different from today's synchrotron sources. The very dense electron pulses necessary for XFEL operation cannot be produced by a circular machine such as a synchrotron, unless the machine is impracticably huge. Therefore the fourth generation sources will rely on high energy linear accelerators (Fig. 2), which can produce much denser electron pulses.

At present XFELs are theoretical, although that could change in the next decade. Existing FELs only emit radiation in the infrared range, but current physics theory can be reasonably extrapolated and detailed plans for two XFELs exist: one in the United States (the Linac Coherent Light Source, LCLS, at the Stanford Linear Accelerator Center, SLAC; Fig. 2)2 and another in Hamburg, Germany (the TeV Energy Superconducting Linear Accelerator, TESLA, at the Deutsches ElektronenSynchrotron, DESY) $)^{3,4}$. While these plans look good on paper, the predicted physics of an XFEL that would emit radiation in the few $\AA$ wavelength range must first be well understood, and some of the basic tenets tested, before building can begin. Such experiments and development of hardware that

g. 1 Peak brightness calculated fo LCLSand for other X-ray sources operating or under construction. Figure courtesy of the Stanford Linear Accerator Center. 


\section{editorial}

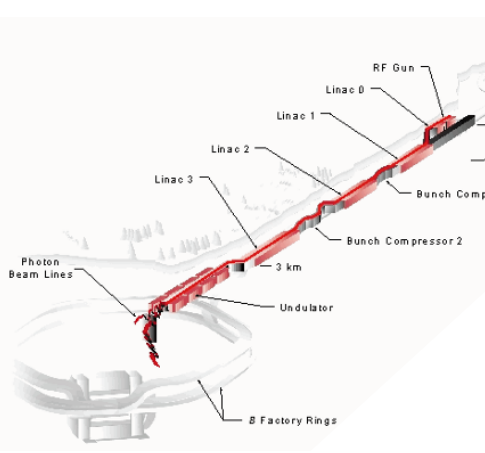

Fig. 2 Concept design graphic of the proposed Linac Coherent Light Source (LCLS), showing the existing linear accelerator (linac) and the proposed extensions to create the XFEL. Figure courtesy of the Stanford Linear Accerator Center.

1. Dexter, M. The Wellcome Trust/ government joint infrastructure funding initiative. (13 July, 1998) http://www.wellcome.ac.uk/ 2. Nuhn, H.-D. Linac coherent light source. (13 July, 1998) http://www-ssrl.slac.stan ford.edu/lds/lds.html

3. Wroblewski, T. A superbrilliant X-ray laser facility. (13 July, 1998) http://www.desy. de/

4. Marx, M. TESLA FEL. (13 July, 1998) http://www-mpy.desy.de/fel/

5. Birgeneau, R.J. (chair) Report of the Basi Energy Sciences Advisory Committee Pane on DOE Synchrotron Radiation Sources and Science. (November, 1997).

6. Chen, P., Tomov, I.V. and Rentzepis, P.M. Time resolved heat propagation in a gold crystal by means of picosecond X-ray diffraction. J. Chem. Phys. 104, 10001-1000 (1997). may be needed (for example, better photocathode guns to release dense electron pulses) is occurring at laboratories around the world.

Recognizing that an XFEL would open up new areas of research across several disciplines, the Basic Energy Sciences Advisory Committee Panel on Department of Energy Synchrotron Radiation Sources and Science (commonly known as the Birgeneau panel since it was headed by Robert Birgeneau of the Massachusetts Institute of Technology) recommended in late 1997 that three million US dollars per year be offered for basic research and design work on FELs ${ }^{5}$. (The same panel also recommended that $\sim 185$ million US dollars per year be allocated for improving US synchrotron sources.) Bill Colson, an FEL expert from the US Naval Postgraduate School in Monterey, California, who served on the Birgeneau panel, notes that this funding will help with the need to demonstrate the practicality of several basic, and necessary, design principles. John Arthur, an X-ray scientist at SLAC, suggests that there are three main reasons why funding FEL research (with an XFEL as an end goal) is reasonable today: (i) accelerator technology for making smaller, more focused electron beams has improved dramatically in recent years; (ii) electron sources (such as photocathode guns) are improving and should soon be capable of generating the very dense pulses of electrons that will be necessary to achieve FEL operation in the X-ray range; and (iii) undulator magnet technology is better understood (largely thanks to the work on synchrotrons) and will facilitate construction of the very long undulator necessary for an XFEL (the LCLS plans call for a $100 \mathrm{~m}$ undulator). If key pilot experiments on small FELs work as expected, then it will be possible to put more faith in the calculations for the large XFEL and funding for an XFEL could be allocated sometime in the next few years. The LCLS is thought by many in the accelerator physics community to be a particularly cost-effective way of extending FEL research into the hard X-ray region. Making use of a portion of the existing Stanford Linear Accelerator the LCLS is estimated to require 100 million US dollars, a fraction of the cost of a new third-generation source such as the APS. Being a testbed for XFEL research, it would initially have only a couple of experimental stations, though it could be upgraded to a multistation user facility in the future.

So, why haven't more biologists heard of the LCLS and TESLA XFEL plans? One answer is that many are still becoming familiar with the big science of accelerators. Use of synchrotrons has become commonplace only over the past five years, and plans for XFELs began to take shape as recently as 1992, when it was realized that technology had improved to the point where the theoretical needs of an XFEL could realistically be met. Another answer is that as exciting as an XFEL sounds - with peak radiation 10 orders of magnitude brighter than that produced by today's third generation sources and a time regime in the fs range - it is not clear that XFELs will be immediately useful for solving biological problems. In contrast, the second and third generation sources currently offer very good realistic prospects for structural biology, and, given that all of these projects are extremely expensive, many biologists would rather see building of additional storage rings and improvement of available facilities to accommodate demand. This is unfortunate for the XFEL research and design community, since biological science is extremely fundable.

To make a strong case for funding, XFEL supporters are routinely asked to identify the kinds of experiments, across a wide range of disciplines, that will be possible with brighter $\mathrm{X}$-ray beams. The key question for biologists is: are there experiments that can be envisioned today that could be performed only with the quality of radiation from an XFEL? With the exceptions of sub-ps time resolved experiments and rapid biological imaging microscopy studies, the answer seems to be that, for now, the second and third generation sources seem more than adequate, especially as they are becoming better tailored to the needs of biologists. Moreover, in the immediate future, improvements at synchrotrons (such as achieving greater coherence of the electron beam and manipulating the time regime using lasers) or in other fields of instrumentation, such as home laboratory X-ray sources driven by optical lasers ${ }^{6}$ (which can have fs time resolutions), may offer more practical promise than an XFEL for currently intractable biological experiments.

The problems faced by scientists today are often ones of prioritization because of time and funding issues. Thus, while admitting that the likely advances in FEL research are exciting, biologists seem personally more concerned with improving access to available brilliant radiation sources, which allow a broad range of biological experiments. While supporting basic research and design work on FELs, the priority for the biological community will likely be to upgrade existing sources, to encourage the building of new synchrotrons equipped for biological applications, and to foster cooperation among facilities to make the most efficient use of beam time, rather than to invest considerable effort into supporting long term plans to build an XFEL. Nevertheless, it would be shortsighted to say that the plans for radically different fourth generation X-ray sources will be of no use to biologists. Did the scientists who developed the first synchrotron particle accelerator sources envision that the X-ray radiation 'by-product' would be critical for future biological advances? Certainly not. Similarly, it is difficult to say definitively that FELs will not be useful to biologists. Improving the technology of X-ray sources could open the doors to as yet unimagined experiments. 